\title{
Footprints of domestication revealed by RAD-tag resequencing in loquat: SNP data reveals a non-significant domestication bottleneck and a single domestication event
}

\author{
Yunsheng Wang ${ }^{1,2}$, Muhammad Qasim Shahid ${ }^{1}$, Shunquan Lin ${ }^{1 *}$, Chengjie Chen ${ }^{1}$ and Chen $\mathrm{Hu}^{1}$
}

\begin{abstract}
Background: The process of crop domestication has long been a major area of research to gain insights into the history of human civilization and to understand the process of evolution. Loquat (Eriobotrya japonica Lindl.) is one of the typical subtropical fruit trees, which was domesticated in China at least 2000 years ago. In the present study, we re-sequenced the genome of nine wild loquat accessions collected from wide geographical range and 10 representative cultivated loquat cultivars by using RAD-tag tacit to exploit the molecular footprints of domestication.

Results: We obtained $26.4 \mathrm{~Gb}$ clean sequencing data from 19 loquat accessions, with an average of $32.64 \mathrm{M}$ reads per genotype. We identified more than 80,000 SNPs distributed throughout the loquat genome. The SNP density and numbers were slightly higher in the wild loquat populations than that in the cultivated populations. All cultivars were clustered together by structure, phylogenetic and PCA analyses.

Conclusion: The modern loquat cultivars have experienced a non-significant genetic bottleneck during domestication, and originated from a single domesticated event. Moreover, our study revealed that Hubei province of China is probably the origin center of cultivated loquat.
\end{abstract}

Keywords: Eriobotrya japonica, Population genomics, Sequencing, SNP marker, Wild loquat

\section{Background}

The domestication of plant and animal is the most important development in the past 13,000 years of human history [1]. The studies on the genetic mechanisms of crop domestication help us to understand the establishment/origin of cultivated species and the history of human civilization, but also offer comprehensive utilization of wild resources to improve the existing varieties and create new germplasm [1-3]. Hence, crop domestication is a very popular topic, and multidisciplinary research methodologies have been used to evaluate domestication

\footnotetext{
* Correspondence: loquat@scau.edu.cn

${ }^{1}$ Key Laboratory of Biology and Germplasm Creation of Horticultural Crop (Southern China), Ministry of Agriculture, South China Agriculrual University, Guangzhou 510642, China

Full list of author information is available at the end of the article
}

[1, 3-5]. In recent decade, research related to crop domestication has been transformed by technologies and discoveries in the genome sciences as well as information-related sciences that are providing new ways for bioinformatics and systems biology [6, 7].

There are six to eight major origin centers of crops in the world and China is the most important one $[8,9]$. In addition to the major crops, such as rice [10], soybean [11] and some other grain crops and vegetables, there are about 52 kinds of fruit crops that were domesticated in China [12]. Among them, the loquat (Eriobotrya japonica Lindl.) is one of the representative subtropical evergreen fruit trees [13]. Loquat is a delicious fruit, rich in amino acid, carbohydrate, fat, cellulose, pectin, carotene, tannin, organic acid, vitamin $\mathrm{A}, \mathrm{B}, \mathrm{C}, \mathrm{B} 1$ and $\mathrm{B} 2$, 
calcium, potassium, phosphorus, iron and other vital elements $[14,15]$. Loquat is also a kind of important traditional Chinese herbal medicine and various parts such as root, stem, leaf, flower and fruit of loquat tree can be used as a medicine for the normal functioning of lungs, arresting cough, anti-inflammatory and strengthening of stomach [16], and also containing some anti-cancer compounds $[17,18]$.

According to the "historical records", one of the greatest books in the Chinese history, loquat trees had been cultivated in the royal gardens for the refreshment of king and princess in the Han Dynasty of China 2000 years ago. In twelfth century, loquat had been introduced into Japan, and then from China or Japan to the rest of the world [13]. Today, there are more than 30 countries planting loquat in the world, and China producing about $80 \%$ loquat of the world, with the annual production of about 1 million tons [19]. In recent years, loquat planting area and yield showed increasing trends because of high economic returns. Many studies on loquat had been executed, including biology [20], molecular phylogeny [21, 22], genetic diversity analysis [23-26], breeding [27], physiology [28, 29], pharmacology [17, 18], and molecular biology [30]. However, domestication of loquat at molecular level has not been discussed.

Single nucleotide polymorphism (SNP) originated from single nucleotide substitute mutation or insertion/ deletion, and it is the most abundant type of variation in the species or genomes [31], and have many utilizations in the field of life sciences, such as molecular genetics, molecular ecology, evolutionary genetics and association analysis [32-35]. In recent years, with the development of high-throughput genome sequencing technologies and the progress in bioinformatics analyses, the whole genome of number of species have been sequenced and re-sequenced. One of the most important achievements of these sequencing works is that SNP markers of these species have been exploited and were used to construct the genetic map for studies of population genomics, phylogeography, ecological genomics and genome-wide association study (GWAS) [36-39].

As for non-model plant without reference genome, new ways of high throughout-sequencing have also been created to exploit SNPs at large scale, and the restriction-site associated DNA tags (RAD) is a famous one. The so-called "Restriction-site Associated DNA" (RAD) method was first described by Miller et al. [40]. The concept is based on acquiring the sequence adjacent to a set of particular restriction enzyme recognition sites. The application of high throughput sequencing technology has allowed significant progress in developing a RAD genotyping platform [41]. Specifically, large volumes of polymorphism data can be generated by applying massive parallel sequencing and multiplexing with
RAD tag libraries [42], which make it widely used technology in population genetic studies [43-47].

In this study, we used RAD-tag tacit sequencing to genotype 10 representative loquat cultivars and nine wild loquat accessions, which collected from different natural habitats. The main aim was to detect the SNPs for whole loquat genome and to explore domestication event of cultivated loquat, such as the geographical origin of cultivated loquat, whether cultivated loquat population is originated from a single- or multi-domesticated events, and whether genetic bottleneck appeared as did in the other crops during domestication.

\section{Results}

\section{Sequencing data}

We obtained a total of $26.4 \mathrm{~Gb}$ clean sequencing data from 620.2 M reads of Illumina Solexa sequencing. The reads of each sample ranged from $14.16 \mathrm{M}$ to $95.07 \mathrm{M}$, with an average of $32.64 \mathrm{M}$ reads. The sequence data of each sample was ranged from 608.68 to $3898.03 \mathrm{Mb}$, with an average sequence data of $1390 \mathrm{Mb}$, and the average read length was 42.56 bases. The quantity and quality of sequencing data obtained from wild groups were lower than that from cultivated groups under the same experimental conditions. However, the sequencing quality scores of 20 (Q20), which represent an error rate of 1 in 100 , with a corresponding call accuracy of $99 \%$, of sequencing data of all samples were more than $98 \%$, indicating that the sequencing was of high quality, and the data quantity and quality fulfill the requirements for population genomics analysis. The mean GC content of sequencing data was $35.12 \%$, which was slightly higher in wild group (35.35\%) than the cultivated group (34.92\%), suggesting that the sequences adjacent to EcoRI restriction enzyme sites had low content of GC sequence (Table 1).

\section{SNP calling and its distribution pattern}

Beside only $2 \mathrm{Mb}$ assembled sequence data of a wild loquat accession, BD_W, which was collected from Badong county of Hubei province, we obtained about 6 $7 \mathrm{Mb}$ assembled sequences for each genotype from other 18 loquat genotypes. We called SNP site according to the following fundamental principle: the homozygous site assembled by at least 10 loquat accessions were sequenced and at least a base variant was found, and we identified a genotype set with 86454 SNPs by this method (Additional file 1: Table S1). We detected about 60000-70000 SNPs in each loquat accession, except about 20000SNPs found in BD_W sample. The distribution density of SNP in the genome of 19 samples was from 8.24 to $10.52 \mathrm{SNPs} / \mathrm{Kb}$ (Table 2), and the average density in wild loquat was higher in wild population than did in cultivated population (i.e. total SNPs per kb: 
Table 1 Summary of the raw data obtained by RAD-tag resequencing

\begin{tabular}{|c|c|c|c|c|c|}
\hline \multirow[t]{2}{*}{ Group } & \multirow{2}{*}{$\begin{array}{l}\text { Genotype } \\
\text { ID }\end{array}$} & \multicolumn{4}{|l|}{ Raw data } \\
\hline & & Reads (M) & Bases (Mb) & GC (\%) & Q20 (\%) \\
\hline \multirow[t]{10}{*}{ Wild } & TS_W & 23.92 & 1028.62 & 35.49 & 99.01 \\
\hline & WF_W & 14.16 & 608.68 & 35.15 & 98.55 \\
\hline & BD_W & 32.03 & 1357.43 & 35.86 & 99.21 \\
\hline & LC_W & 16.11 & 676.79 & 35.01 & 98.91 \\
\hline & SM_W & 17.91 & 788.21 & 35.25 & 98.49 \\
\hline & MT_W & 18.08 & 741.38 & 36.96 & 98.77 \\
\hline & BJ_W & 33.70 & 1381.59 & 34.66 & 98.66 \\
\hline & AL_W & 33.19 & 1460.43 & 34.98 & 99.01 \\
\hline & HZ_W & 57.80 & 2369.91 & 34.76 & 98.95 \\
\hline & Average & 27.43 & 1157.00 & 35.35 & 98.84 \\
\hline \multirow[t]{11}{*}{ Cultivated } & DWX_C & 24.82 & 1067.40 & 34.96 & 98.89 \\
\hline & BY_C & 34.57 & 1486.72 & 35.09 & 99.09 \\
\hline & YN_C & 32.03 & 1441.15 & 35.03 & 99.11 \\
\hline & SJPP_C & 23.76 & 1021.64 & 34.97 & 99.14 \\
\hline & RTBS_C & 67.82 & 2994.30 & 34.72 & 98.20 \\
\hline & ZZ6H_C & 23.42 & 1030.38 & 35.00 & 99.17 \\
\hline & MM_C & 95.07 & 3898.03 & 34.68 & 98.90 \\
\hline & MBC_C & 27.61 & 1214.65 & 35.06 & 99.13 \\
\hline & Biano_C & 23.10 & 947.06 & 34.79 & 99.08 \\
\hline & Algeria_C & 21.10 & 886.33 & 34.90 & 99.19 \\
\hline & Average & 37.33 & 1598.77 & 34.92 & 98.99 \\
\hline Total Mean & & 32.64 & 1389.51 & 35.12 & 98.92 \\
\hline
\end{tabular}

See Table 6 for genotypes detail

10.16 vs 10.04 (2.4\%), heterozygous SNPs per Kb: 1.99 vs $2.01(-1.0 \%)$, and homozygous SNPs per Kb: 8.16 vs 7.93 $(2.9 \%)$ were detected in wild and cultivated populations, respectively)). However, $t$-test of independent samples on the frequency of total number of SNPs, homozygous SNPs and heterozygous SNPs exhibited a nonsignificant differences between cultivated and wild loquat groups $(\mathrm{P}>0.05)$. We further obtained a SNP data set composed of 6406 SNPs with no gap from whole loquat population (Additional file 1: Table S2). Of these, 4807 and 1599 sites were SNPs and fixed in wild loquat population, while 4231 and 2175 sites were SNPs and fixed in cultivated loquat population, respectively. The ratio of total number of SNPs, with no gap, was $13.61 \%$ higher in wild loquat population than cultivated loquat population (Table 3).

Population genetic structure and genetic differentiation AMOVA analysis of 19 loquat genotypes, based on the SNP data with no gap, revealed that the variance components among populations, among individuals within populations, and within individuals were $14.45 \%, 25.62 \%$, and $59.93 \%$ of the total genetic variance, respectively (Table 4). The results showed that the heterozygosity within loquat individuals accounted for most of the genetic diversity of whole loquat population. The pairwise fixation index, $F_{\mathrm{ST}}$, was 0.16 , which was estimated from pairwise comparison between wild and cultivated groups and was significantly different from zero $(P<0.001)$. These results showed that highly significant genetic differentiation happened between wild and cultivated loquat populations (Table 5).

\section{Phylogenetic analysis}

Unrooted phylogenetic tree of 19 samples showed that nine samples of wild group clustered together at one end of the tree, and 10 cultivated samples were clustered at the other end of the tree (Fig. 1). In the wild group, three samples from Bijie county, Anlong county, and Meitan county, Guizhou province clustered into close proximity to each other, and the sample from Shimian county, Sichuan province was also close to the aforementioned accessions, indicating that the genetic distance of wild loquat population had some regional relationships. However, the wild samples from Hubei and Shaanxi province were more close to cultivated loquat samples and the closest one was TS_W. Interestingly, the cultivar, Sijipipa, exhibited the closest relationships with the wild loquat population, and the cultivars Dawuxing, Yunan and Biano were clustered far from the wild loquat population.

\section{PCA analysis}

The results of principal components analysis of the 19 genotypes showed that the first principal component could explain $5.09 \%$ genetic variation of whole loquat population, and the second principal components can explain $3.38 \%$ genetic variation of whole loquat population (Fig. 2). The first and the second principal components could clearly separate wild and cultivated loquats, and the results showed that the wild and cultivated loquat have entirely different evolutionary trends.

\section{Population structure}

Population structure helps us to understand the evolutionary process of a population or species through the association between genotype and phenotype, and to determine the groups or subgroups of different individuals/populations. When $\mathrm{k}$ was set as $2, \mathrm{Al}$-W, MT_W, BJ_W, SM_W, LC_W and BD_W were clustered into one wild group, and nine cultivated loquat cultivars were clustered into cultivated group, and HZ_W, WF_W, TS_W and SJPP_C were assigned to aforementioned two groups. However, HZ_W, WF_W and TS_W have a higher probability to be included in wild group, and the SJPP_C has a higher probability to join cultivated group. When $\mathrm{k}$ was set as 3, Al_W, MT_W and BJ_W from 
Table 2 Summary of the SNPs with a genotyping rate of homozygous sites less than $50 \%$

\begin{tabular}{|c|c|c|c|c|c|c|c|c|}
\hline Group & $\begin{array}{l}\text { Genotype } \\
\text { ID }\end{array}$ & $\begin{array}{l}\text { Assembled } \\
\text { sequence (bp) }\end{array}$ & $\begin{array}{l}\text { Total } \\
\text { SNPs }\end{array}$ & $\begin{array}{l}\text { Heterozygous } \\
\text { SNPs }\end{array}$ & $\begin{array}{l}\text { Homozygous } \\
\text { SNPs }\end{array}$ & $\begin{array}{l}\text { Total SNPs } \\
\text { per kb }\end{array}$ & $\begin{array}{l}\text { Heterozygous SNPs } \\
\text { number per Kb }\end{array}$ & $\begin{array}{l}\text { Homozygous SNPs } \\
\text { per Kb }\end{array}$ \\
\hline \multirow[t]{10}{*}{ Wild } & AL_W & 7645931 & 77745 & 15124 & 62621 & 10.17 & 1.98 & 8.19 \\
\hline & BD_W & 2107974 & 20324 & 2441 & 17883 & 9.64 & 1.16 & 8.48 \\
\hline & BJ_W & 6828806 & 71837 & 13911 & 57926 & 10.52 & 2.04 & 8.48 \\
\hline & HZ_W & 7063593 & 72665 & 12810 & 59855 & 10.29 & 1.81 & 8.47 \\
\hline & LC_W & 6755448 & 69772 & 14319 & 55453 & 10.33 & 2.12 & 8.21 \\
\hline & MT_W & 6693879 & 69792 & 15421 & 54371 & 10.43 & 2.30 & 8.12 \\
\hline & SM_W & 7406774 & 72054 & 18028 & 54026 & 9.73 & 2.43 & 7.29 \\
\hline & TS_W & 7802112 & 78502 & 16920 & 61582 & 10.06 & 2.17 & 7.89 \\
\hline & WF_W & 7105355 & 72840 & 13639 & 59201 & 10.25 & 1.92 & 8.33 \\
\hline & Average & 6601097 & 672812 & 13624 & 53658 & 10.16 & 1.99 & 8.16 \\
\hline \multirow[t]{11}{*}{ Cultivated } & Algeria_C & 6734550 & 69033 & 17950 & 51083 & 10.25 & 2.67 & 7.59 \\
\hline & Biano_C & 6378374 & 65886 & 13780 & 52106 & 10.33 & 2.16 & 8.17 \\
\hline & BY_C & 7509796 & 76407 & 15521 & 60886 & 10.17 & 2.07 & 8.11 \\
\hline & DWX_C & 7191808 & 74136 & 13487 & 60649 & 10.31 & 1.88 & 8.43 \\
\hline & MBC_C & 7473459 & 74392 & 17565 & 56827 & 9.95 & 2.35 & 7.60 \\
\hline & MM_C & 7170711 & 73359 & 18597 & 54762 & 10.23 & 2.60 & 7.64 \\
\hline & RTBS_C & 6740816 & 55532 & 2993 & 52539 & 8.24 & 0.44 & 7.79 \\
\hline & SJPP_C & 7199676 & 73112 & 15336 & 57776 & 10.15 & 2.13 & 8.02 \\
\hline & YN_C & 7668847 & 75122 & 13710 & 61412 & 9.80 & 1.79 & 8.01 \\
\hline & ZZ6H_C & 7310710 & 72836 & 14474 & 58362 & 9.96 & 1.98 & 7.98 \\
\hline & Average & 7137875 & 70982 & 14341 & 56640 & 9.94 & 2.01 & 7.93 \\
\hline Total & Mean & 6883612 & 69229 & 14001 & 55227 & 10.04 & 2.00 & 8.04 \\
\hline
\end{tabular}

See Table 6 for genotypes detail

Guizhou province constituted a group, MBC_C, Biano_C, YN_C and DWX_C were clustered into one group, and BD_W, HZ_W, WF_W TS_W and SJPP_C were assembled into another group, while SM_W, LC_W, BY_C, ZZ6H_C, MM_C and Algeria_C were clustered into two separate groups. When $\mathrm{k}$ was set as 4, three samples collected from Guizhou province were further divided into two groups, and the clustering pattern of other samples was almost consistent with $\mathrm{K}=3$; When $\mathrm{k}$ was set as 5 , the wild samples were divided into three groups with three samples in each group, and the cultivated loquat cultivars was clustered into two groups (Fig. 3).

Table 3 Summary of SNPs from the genotyping data of all 19 loquat accessions with no gap

\begin{tabular}{lll}
\hline Data ID & $\begin{array}{l}\text { Number of } \\
\text { total SNPs }\end{array}$ & $\begin{array}{l}\text { Number of } \\
\text { fixed sites }\end{array}$ \\
\hline Whole loquat population & 6406 & 0 \\
Wild population & 4807 & 1599 \\
Cultivated population & 4231 & 2175 \\
\hline
\end{tabular}

\section{Discussion}

The representativeness of samples

According to the extensive survey, the wild loquat (Eriobotrya japonica Lindl.) populations have been found in Hubei, Sichuan, Yunnan, Guizhou, Guangxi and Guangdong provinces of China. Among them, Hubei and Guizhou provinces are the major distributing areas of wild loquat [48]. In the present study, a total of 9 wild accessions and 10 loquat cultivars were selected for sequencing. Among 9 wild loquat samples, four samples were collected from different regions of

Table 4 Analysis of molecular variance (AMOVA) of 19 loquat genotypes

\begin{tabular}{lrcl}
\hline $\begin{array}{l}\text { Source of } \\
\text { variation }\end{array}$ & $\begin{array}{l}\text { Sum of } \\
\text { squares }\end{array}$ & $\begin{array}{l}\text { Variance } \\
\text { components }\end{array}$ & $\begin{array}{l}\text { Percentage } \\
\text { variation }\end{array}$ \\
\hline $\begin{array}{l}\text { Among } \\
\text { populations }\end{array}$ & 3347.063 & 125.63590 & 14.44924 \\
$\begin{array}{l}\text { Within } \\
\text { populations }\end{array}$ & 16432.094 & 222.73111 & 25.61604 \\
$\begin{array}{l}\text { Within } \\
\text { individuals }\end{array}$ & 9901.500 & 521.13158 & 59.93472 \\
Total & 29680.658 & 869.49858 & \\
\hline
\end{tabular}


Table 5 Genetic differentiation between wild and cultivated populations

\begin{tabular}{lll}
\hline Pairwise index $\left(F_{\text {ST }}\right)$ & Wild population & $\begin{array}{l}\text { Cultivated } \\
\text { population }\end{array}$ \\
\hline Wild population & - & $* * *$ \\
Cultivated population & 0.15955 & - \\
\hline
\end{tabular}

Hubei province, three samples from different regions of Guizhou province, one from Sichuan province, and one from Shanxi province. All these nine samples were regional representatives of species distribution range and separated from each other by far geographical distance. Among 10 loquat cultivars, six are the main cultivars of different loquat producing areas of China, such as Zhejiang province, Fujian province, Jiangsu province, Sichuan province, Yunnan province and Guangdong province, and other four are the main cultivars of Japan, Italy, Spain and the United States of America. Molecular

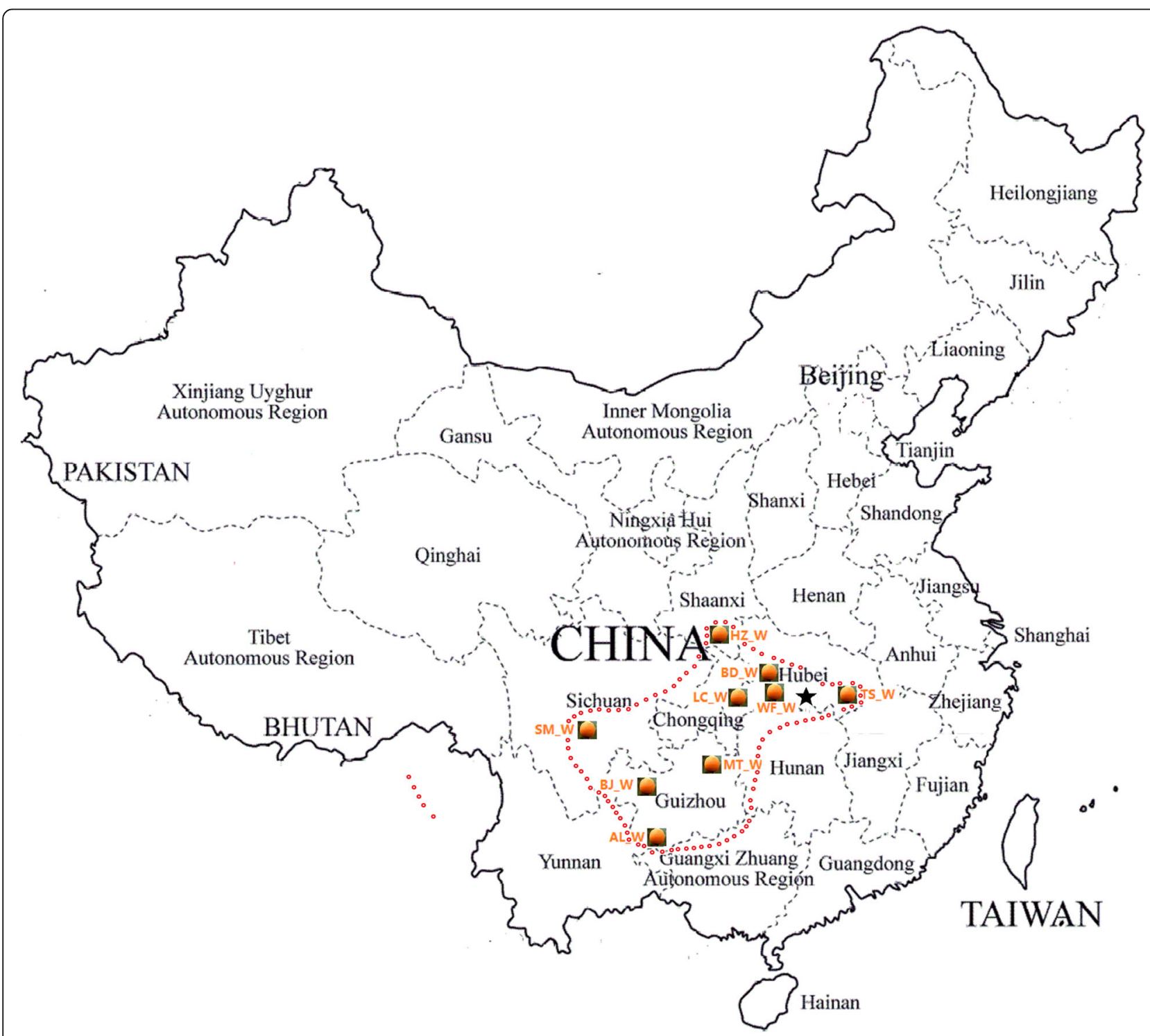

Fig. 1 Phylogenetic tree of 19 loquat genotypes was constructed based on neighbor-joining (bootstrap value $=500$ ) method. AL_W, BJ_W, MT_W, SM_W, HZ_W, BD_W, LC_W, WF_W, TS_W represent the wild loquat genotypes; AL_W, BJ_W and MT_W were collected from Anlong, Bijie and Meitan counties of Guizhou province, SM_W was collected from Simian county of Sichuan province, HZ_W was collected from Hanzhong county of Shanaxi province, BD_W, LC_W, WF_W, TS_W were collected from Badong, Lichuan, Wufeng and Tongshan counties of Hubei province, respectively; DWX_C, BY_C, YN_C, SJPP_C, RTBS_C, ZZ6H_C, MM_C, MBC_C, Biano_C, Algeria_C are all loquat cultivars, and known as Dawuxing, Baiyu, Yunan, Sijipipa, Ruantiaobaisha, Zaozhong No.6, Mogi, MBC, Biano and Algeria, respectively 


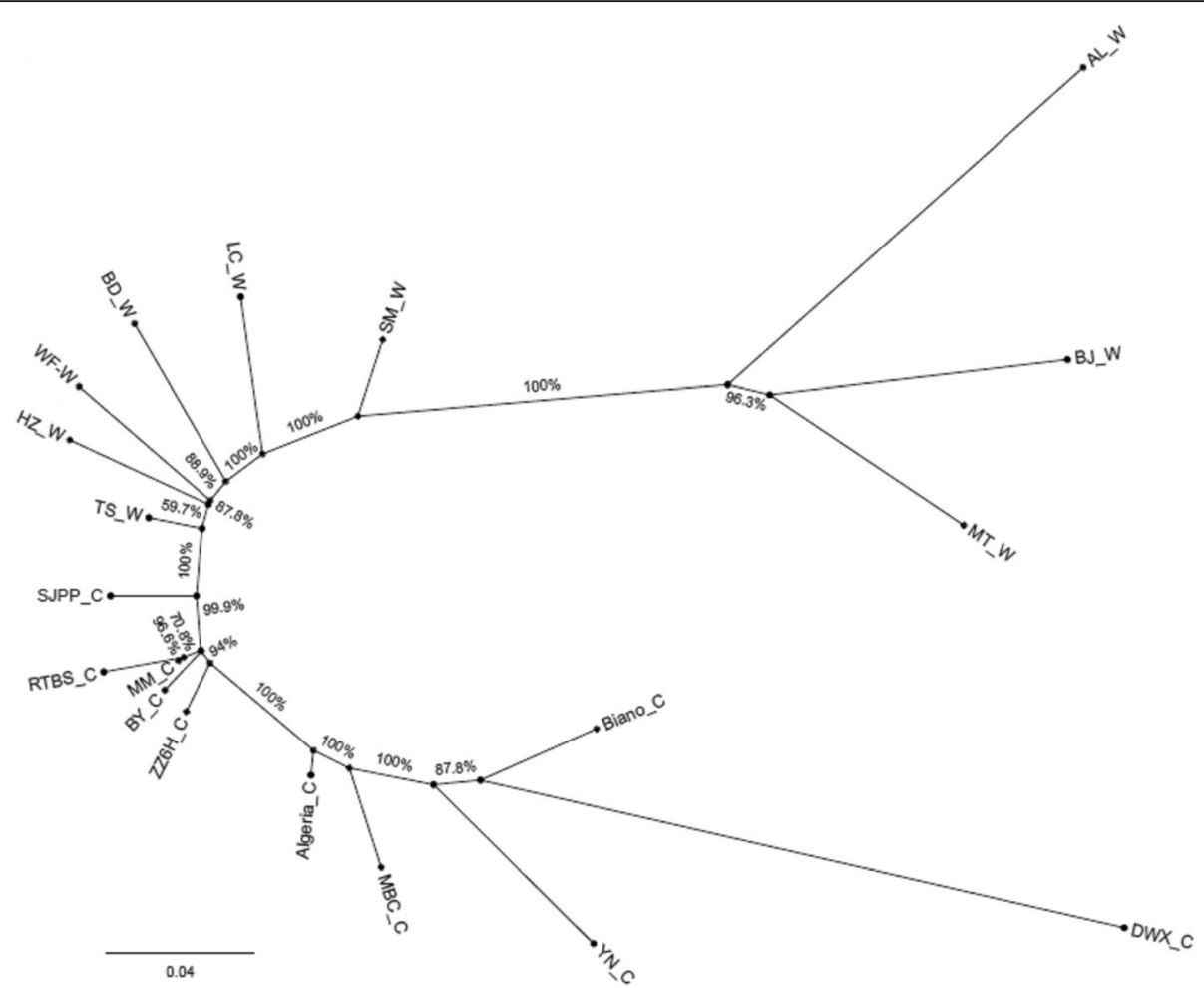

Fig. 2 Principal component analysis (PCA) of 19 loquat genotypes. AL_W, BJ_W, MT_W, SM_W, HZ_W, BD_W, LC_W, WF_W, TS_W represent the wild loquat genotypes; AL_W, BJ_W and MT_W were collected from Anlong, Bijie and Meitan counties of Guizhou province, SM_W was collected from Simian county of Sichuan province, HZ_W was collected from Hanzhong county of Shanaxi province, BD_W, LC_W, WF_W, TS_W were collected from Badong, Lichuan, Wufeng and Tongshan counties of Hubei province, respectively; DWX_C, BY_C, YN_C, SJPP_C, RTBS_C, ZZ6H_C, MM_C, MBC_C, Biano_C, Algeria_C are all loquat cultivars, and known as Dawuxing, Baiyu, Yunan, Sijipipa, Ruantiaobaisha, Zaozhong No.6, Mogi, $M B C$, Biano and Algeria, respectively

pedigree analysis showed that those cultivars almost represent different genetic clusters of current loquat cultivars around the world $[25,26]$.

\section{Genetic bottleneck in cultivated loquat during domestication}

In the process of crop domestication, the genetic bottleneck is common, which indicate that the effective size of crops population were significantly fewer than its corresponding wild progenitor population [49]. Compared to the nearest wild progenitors, crops population appeared to have lower genetic diversity, higher level of linkage disequilibrium and lower SNP frequencies, such as rice $[38,39]$, soybean $[11,50]$, cucumber [51], peach [52], and tomato [53]. However, there was no clear genetic bottleneck in several crops, such as grape [54]. In this study, we found that the SNP frequencies in wild loquat population were only $2.4 \%$ higher than that in cultivated loquat population. Meanwhile, AMOVA analysis showed that $85.55 \%$ of genetic variants existed among- and inter-individuals, while only $14.45 \%$ happened between wild and cultivated populations. The independent samples $t$-test exhibited non-significant differences between cultivated and wild loquat groups $(P>0.05)$ for the frequency of total number of SNPs, homozygous SNPs and heterozygous SNPs. Above all results suggested that a non-significant genetic bottleneck appeared during the domestication of loquat. We deduced following major reasons for these results: 1) the domestication history of loquat is not so long; 2) loquat domestication is not yet complete, because there are non-significant differences for physiological and morphological traits between cultivated and wild loquat except that the fruit size of cultivated loquat is bigger than that of wild loquat.

\section{The origin center of cultivated loquat}

Discovering the origin center of a crop is not only an interesting scientific question, but also an important social science issue, because the ancient civilization of mankind is actually a farming civilization, and the action of crops domestication represents the height of the agricultural civilization to a certain extent. In fact, most crops were thought to have originated in the more developed areas of ancient civilizations [2, 9]. The valley of Qingshuijiang river of South-West Hubei province or valley of Daduihe river of South-West Sichuan province have 


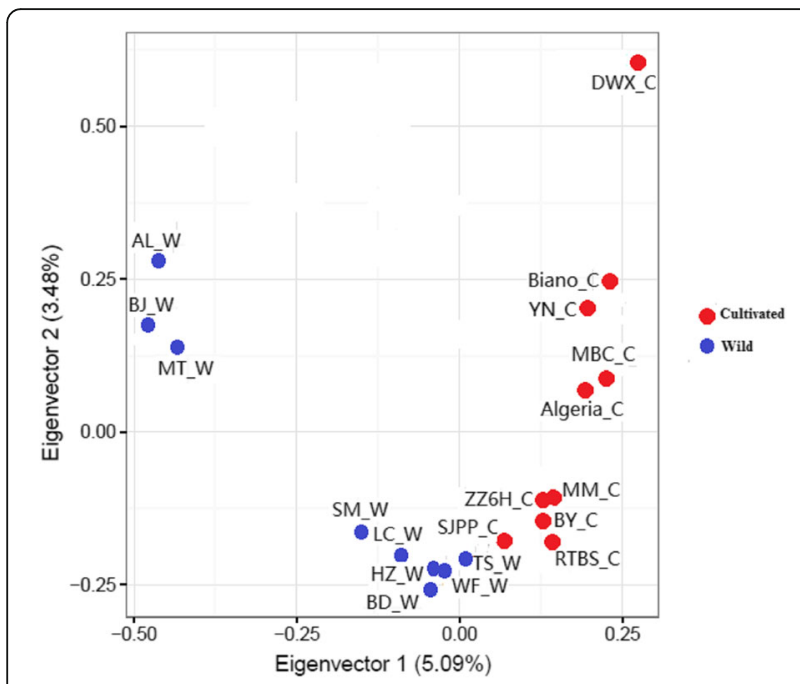

Fig. 3 Population structure analysis of 19 loquat genotypes. $X$ - and $Y$ axis are representing genotypes and probability levels, respectively. AL_W, BJ_W, MT_W, SM_W, HZ_W, BD_W, LC_W, WF_W, TS_W represent the wild loquat genotypes; AL_W, BJ_W and MT_W were collected from Anlong, Bijie and Meitan counties of Guizhou province, SM_W was collected from Simian county of Sichuan province, HZ_W was collected from Hanzhong county of Shanaxi province, BD_W, LC_W, WF_W, TS_W were collected from Badong, Lichuan, Wufeng and Tongshan counties of Hubei province, respectively; DWX_C, BY_C, YN_C, SJPP_C, RTBS_C, ZZ6H_C, MM_C, MBC_C, Biano_C, Algeria_C are all loquat cultivars, and known as Dawuxing, Baiyu, Yunan, Sijipipa, Ruantiaobaisha, Zaozhong No.6, Mogi, MBC, Biano and Algeria, respectively

been inferred as the original place of cultivated loquat according to the field investigations of morphological traits and geographical distribution $[55,56]$. In this study, the sample, TS_W, collected from Hubei province was the most closest to the cultivated loquat according to the phylogenetic tree. Structure analysis also showed that this genotype was clustered with some cultivated loquat cultivars, such as SJPP_C (K=2,3, and 4). However, the wild sample, SM_W from Sichuan province, was far from the cultivated loquat based on above mentioned analysis. According to the archaeological work, in the 1970s, when people dug an ancient tomb that was constructed in Han dynasty, which is located in Jiangling county of Hubei province and near to Tongshan county, the carbonization relic of loquat seed was found among many funeral items, and this was the earliest archaeological evidence of loquat cultivation [13]. So, based on molecular and archaeological evidence, we deduced that the Hubei province, not Sichuan province, might be the origin center of cultivated loquat.

\section{A single domestication origin of cultivated loquat}

A crop that comes from a single domestication event or multiple domestication events is often present dispute based on the ancient book record and archaeology, which was used in corresponding research in the past
[57-59]. However, modern molecular phylogeny, molecular population genetics and phylogeographical studies showed that most crops were evolved from single domesticated event, such as einkorn wheat [60], potato [61], soybean [50], maize [62], japonica rice [10], and cucumber [63]. Multi-domesticated events were not so common and it was observed in common bean [64] and barley [65].

China has about 2000 years long history for loquat cultivation until the twelfth century. Loquat was introduced into Japan from China and then from China and Japan to other parts of the world [19]. In the process of artificial selection and ecological adaptability, cultivated loquats differentiated into different cultivars or strains. Liu et al. [66] revealed three groups of cultivated loquat by analyzing 100 morphological and biochemical traits. Cultivated loquat of China was divided into two categories, namely as subtropical and tropical groups according to the regional ecological adaptability [67]. Two ecological groups of cultivated loquat were found according to the plant morphology such as leaf color, fruit size, and sugar content [48]. These analyses suggested that cultivated loquat may have been domesticated at multiple places, or may have been domesticated at just one place, and then spread outside along different routes, and differentiated into different groups. In the present study, structure, phylogenetic and PCA analyses have

Table 6 Names and geographical origin of loquat genotypes used in the study

\begin{tabular}{lll}
\hline Code & Genotype & Geographical origin \\
\hline DWX_C & Dawuxing & Sichuan province, China \\
BY_C & Baiyu & Jiangsu province, China \\
YN_C & Younan & Guangdong province, China \\
SJPP_C & Sijipipa & Yunnan province, China \\
RTBS_C & Ruantiao baisha & Zhejiang province, China \\
ZZ6H_C & Zaozhong No.6 & Fujian province \\
Biano_C & Biano & Italy \\
MBC_C & MBC & America \\
MM_C & Mogi & Japan \\
Algeria_C & Algeria & Spain \\
TS_W & Wild loquat & Tongshan county, Hubei province \\
WF-W & Wild loquat & Wufeng county, Hubei province \\
BD_W & Wild loquat & Badong county, Hubei province \\
LC_W & Wild loquat & Lichuan county, Hubei province \\
MT_W & Wild loquat & Meitan county, Guizhou province \\
BJ_W & Wild loquat & Bijie county, Guizhou province \\
AL_W & Wild loquat & Anlong county, Guizhou province \\
SM_W & Wild loquat & Shimian county, Sichuan province \\
HZ_W & Wild loquat & Luding county, Sichuan province \\
\hline
\end{tabular}


shown that all the cultivated loquat samples were clustered into the same group, which showed that cultivated loquat samples exhibited mono-phylogenic origin. So, we concluded that cultivated loquat was originated from a single domestication event. However, cultivated loquat cultivars exhibited differentiation by structure analysis $(k=5)$. One group, SJPP_C, RTBS_C, BY_C, ZZ6H_C and MM_C, might be comprised of early domesticated cultivars, and another group involving Biano_C, YN_C and DWX_C might be domesticated recently, while Algeria_C and $\mathrm{MBC} \_\mathrm{C}$ clustered between these two groups.

\section{Conclusions}

Here, we re-sequenced the genome of nine wild loquat accessions collected from wide geographical range and 10 representative cultivated loquat cultivars by using RADtag tacit to exploit the molecular footprints of domestication. The results showed that the SNP density and numbers were non-significantly higher in the wild loquat populations than that in the cultivated populations. All cultivars were clustered together by structure, phylogenetic and PCA analyses. The modern loquat cultivars have experienced a non-significant genetic bottleneck during domestication, and originated from a single domesticated event. Moreover, our results revealed that Hubei province of China might be the origin center of cultivated loquat.

\section{Methods}

\section{Plant sampling and DNA extraction}

The leaves of ten cultivated loquat cultivars were sampled from Horticultural germplasm conversation center of South China Agricultural University (SCAU), where most of the major cultivated loquat cultivars of the world have been planted, and nine wild loquats were sampled from native wild loquat populations representing their natural distribution range (Table 6; Fig. 4). The DNA was extracted according to CTAB method [68].
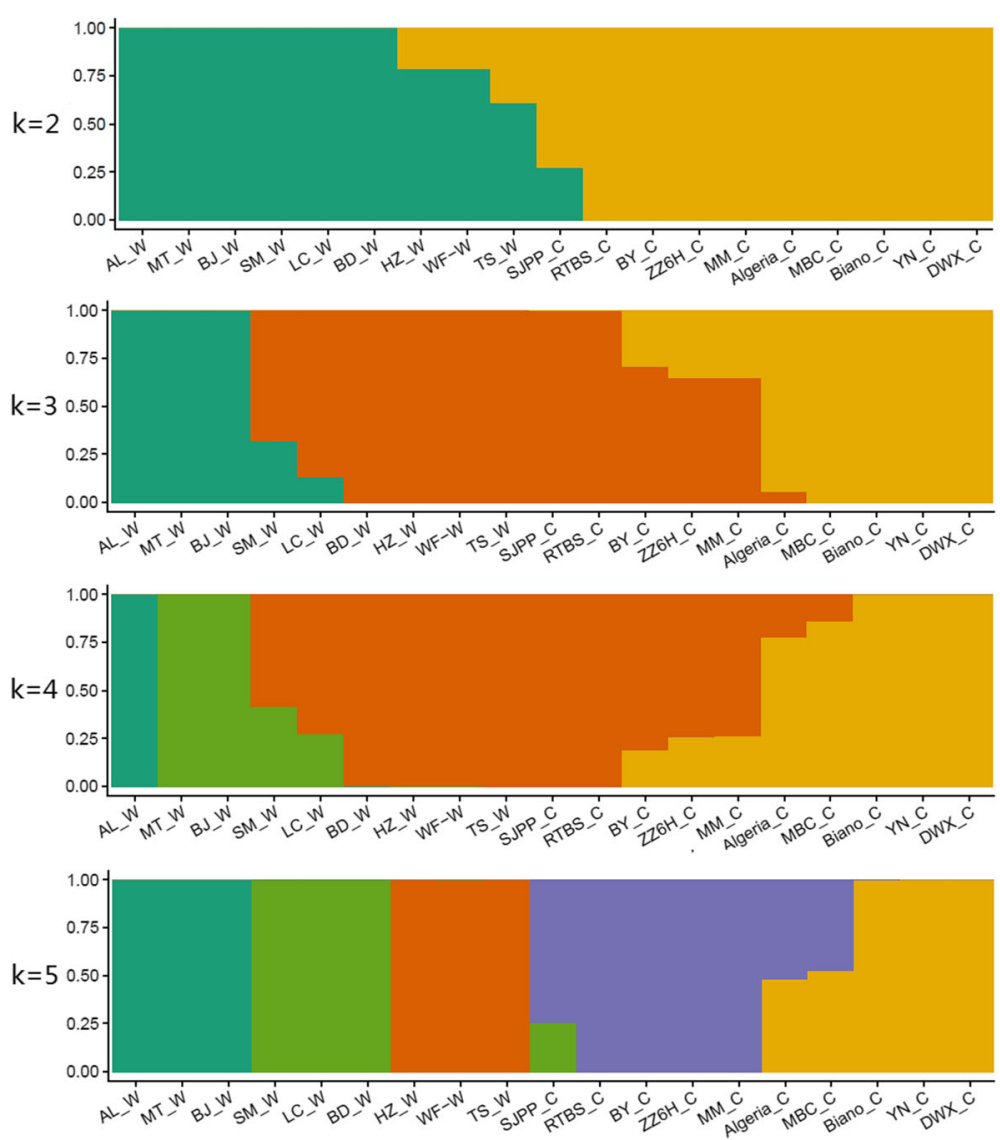

Fig. 4 The sampling location of wild loquat genotypes. Orange levels (loquat fruit) represent the sampling sites of wild loquat genotypes, star (black) represents the position of earliest archaeological site where carbonation relic of loquat seed was found, and the area inside the red circles is natural habitat of wild loquat. AL_W, BJ_W and MT_W were collected from Anlong, Bijie and Meitan counties of Guizhou province, SM_W was collected from Simian county of Sichuan province, HZ_WW was collected from Hanzhong county of Shanaxi province, BD_W, LC_W, WF_W, TS_W were collected from Badong, Lichuan, Wufeng and Tongshan counties of Hubei province, respectively 


\section{The construction of RAD-tag libraries}

Mixed library of RAD-tag (restriction-site associated DNA tags) was constructed in this study by the protocols described by Etter et al. [69], and it is as follows: (A) Restriction enzyme EcoRI digested about $1 \mu \mathrm{g}$ genomic DNA and joint P1 adapter ((P1-FOR-xxxxx: ' -Phos- AATGATACG GCGACCACCGAGATCTACACTTTCCCTACACGACG CTCTTCCGATCTXxxxxTGC*A-3 ' P1-REV-xxxxx: 5 ' -Phos-xxxxxAGATCGGAAGAGCGTCGTGTAGGGAAA GAGTGTAGA; TCTCGGTGGTCGCCGTATCAT*T-3 ') which contains sequences of primers P1-PCR (5 '-AATG ATACGGCCCACCGA-3 ') for amplification, primer binding sites in Illumina Genome Analyzer and short tags to distinguish samples; (B) Samples with different adapters were mixed to be broken into segments of $300 \sim 700 \mathrm{bp}$ using physical method; (C) Joint P2 adapter (P2-FOR:' -Phos-GATCGGAAGAGCGGTTCAGCAGGAATGCCG AGACCGATCAGAACAA-3 'P2-PE-REV: 5' -Phos CA AG CAGAAGACGGCATACGAGATCGGTCTCGGCAT TCCTGCTGAACCGCTCTTCCG ATC*T-3 ', including primer sequences: P2-PCR, 5 ' -AATGATACGGCGACCA CCGA-3 '), recovery; (D) PCR: 5-10 rounds of amplification target sequence (enrichment and sequencing primers for P1-PCR $\backslash$ P2-PCR).

\section{Sequencing, raw data processing and SNP genotyping}

Illumina Solexa sequencing was done by sequencer Hiseq 2000 , and sequencing type was SE50. We make sure that clean data were at least more than $0.6 \mathrm{G}(0.8 \times \mathrm{C})$ for each individual. After sequencing, the raw data were processed in three steps: first, we allocated the raw data to its own origin sample according to the labels $(4 \sim 8 \mathrm{bp})$ used for sequencing to distinguish samples ( $4 \sim 8 \mathrm{bp})$; secondly, we filtered label sequences and the joint to exclude the pollution; thirdly, we discard those raw data with low quality base $(\mathrm{Q} \leq 5(\mathrm{E}))$ number accounted for more than half of the whole reads.

The rest clean and high quality raw data were used for further SNPs calling by software Stacks (http://catchenlab.life.illinois.edu/stacks). Software Stacks uses shortread sequence data to identify and genotype loci in a set of individuals either de novo or by comparing to a reference genome. From reduced representation Illumina sequence data, such as RAD-tags, Stacks can recover thousands of single nucleotide polymorphism (SNP) markers useful for the genetic analysis of crosses or populations [70]. We used SPSS 24.0 (https://spss.en.softonic.com/) to execute the significance test of difference in average frequency of total SNPs, homozygous SNPs and heterozygous SNPs between wild and cultivated loquat by using $T$ test of independent samples. For further analysis, we filtered the genotype data with the loss rate less than $50 \%$ (Additional file 1: Table S1) or with no loss (Additional file 1: Table S2).

\section{Population genetics and evolutionary analysis}

We used Arliquin 3.11 software [71] to execute the AMOVA and genetic differentiation analysis using SNP dataset with no loss/gap (Additional file 1: Table S2). Neighbor-Joining method [72, 73] was employed to construct the phylogenic relationships between loquat genotypes based on SNP dataset with no loss/gap (Additional file 1: Table S2) by using program MAGE 5.0 [74], and removed all the ambiguous positions for each sequence pair and set bootstrap $=500$. The PCA analysis was executed by software EIGENSOFT 3.0 [75] using SNP dataset with the loss rate less than $50 \%$ (Additional file 1: Table S1). The software Structure v2.3.4 [76] was used to analyze the genetic structure using SNP dataset with the loss rate less than 50\% (Additional file 1: Table S1) with admixture model, and $\mathrm{K}$ was set from 2 to 6 .

\section{Additional file}

Additional file 1: Table S1. SNP dataset with less than $50 \%$ loss. Table S2.

SNP dataset with no gap. (XLS $27844 \mathrm{~kb}$ )

Abbreviations

PCA: Principal component analysis; SNP: Single nucleotide polymorphism

Acknowledgements

The authors thank to lab members for assistance.

Funding

This research was supported by National Natural Science Foundation of China (31560091), the Key project of the Education Department of Guizhou Province [KY(2013)186], and Key joint fund of the Kaili University and Science and Technology Department of Guizhou Province [LH(2015)7754].

Availability of data and materials

Sequencing data and UCE fasta files are available from the NCBI SRA: SRP104011 (BioProject: 2056770; PRJNA: 352131).

\section{Authors' contributions}

W.Y., and L.S., designed the research. W.Y., M.Q.S., and H.C., performed the experiment. W.Y., M.Q.S., L.S., and C.C., analyzed the data. The article was written by W.Y., and M.Q.S., with additional comments from L.S., C.C., and H.C. All authors read and approved the final manuscript.

\section{Competing interests}

The authors declare that they have no competing interests.

Consent for publication

Not applicable.

Ethics approval and consent to participate Not applicable.

\section{Publisher's Note}

Springer Nature remains neutral with regard to jurisdictional claims in published maps and institutional affiliations.

\section{Author details}

'Key Laboratory of Biology and Germplasm Creation of Horticultural Crop (Southern China), Ministry of Agriculture, South China Agriculrual University, Guangzhou 510642, China. ${ }^{2}$ College of Environment and Life Science, Kaili University, Guizhou 556011, China. 


\section{Received: 6 December 2016 Accepted: 27 April 2017}

\section{Published online: 06 May 2017}

\section{References}

1. Diamond J. Evolution, consequences and future of plant and animal domestication. Nature. 2002:418:700-7.

2. Olsen KM, Gross BL. Detecting multiple origins of domesticated crops. Proc Natl Acad Sci U S A. 2008;5:13701-2.

3. Wang YS, Wang Y, Huang HW. Genetics research into crop domestication and its application in soybean breeding. Chinese Bull Bot. 2008;25(2):221-9.

4. Burger JC, Chapman MA, Burke JM. Molecular insights into the evolution of crop plants. Am J Bot. 2008;95:113-22.

5. Ross-lbarra J, Morell PL, Gaut BS. Plant domestication, a unique opportunity to identify the genetic basis of adaptation. Proc Natl Acad Sci U S A. 2007; 104:8641-8.

6. Vaughan DA, Balazs E, Heslop-Harrison JS. From Crop Domestication to Super-domestication. Ann Bot. 2007;100:893-901.

7. Henry RJ. Next-generation sequencing for understanding and accelerating crop domestication. Brief Funct Genomics. 2011;15(5):1-6.

8. Hawkes JG. The diversity of crop plants. Cambridge: Harvard University Press; 1983. p. 358-66.

9. Vavilov NI. Origin and geography of cultivated plants. Cambridge: Cambridge University Press; 1992.

10. Huang X, Kurata N, Wei X, Wang ZX, Wang A, Zhao Q. A map of rice genome variation reveals the origin of cultivated rice. Nature. 2012; 490(7421):497-501.

11. Guo J, Wang $Y$, Song $C$, Jiang $X$, Wang $L$, Wang $X$, et al. A single origin and moderate bottleneck during domestication of soybean (Glycine max): implications from microsatellites and nucleotide sequences. Ann Bot. 2010;106:505-14.

12. Qu ZZ, Sun YW. Viewpoint on species of fruit tree. Beijing: Agriculture Press; 1990.

13. Lin S, Sharpe RH, Janick J. Loquat: Botany and Horticulture. Hort Rev. 1999; 23:233-76.

14. Hasegawa PN, Faria AF, Mercadante AZ. Chemical composition of five loquat cultivars planted in Brazil. Food Sci Technol (Campinas). 2010; 30(2):552-9.

15. Piva G, D'Asaro A, Fretto S, Farina V, Mazzaglia A. Chemical and sensory characteristics of five loquat cultivars. Acta Hort. 2015;1092:167-71.

16. Maher K, Yassine BA, Sofiane B. Anti-inflammatory and antioxidant properties of Eriobotrya japonica leaves extracts. Afr Health Sci. 2015;15(2): 613-20.

17. Ito H, Kobayashi E, Takamatsu Y, Li S, Hatano T, Sakagami H, et al. Polyphenols from Eribotrya japonica and their cytotoxicity against human oral cancer cell lines. Chem Pharmaceutical Bull. 2000;48:687-93.

18. Yuan Y, Gao Y, Song G, Lin S. Ursolic acid and oleanolic acid from Eriobotrya fragrans inhibited the viability of A549 cells. Natural Product Comm. 2015; 10(2):239-42.

19. Lin S, Huang $X$, Cuevas J, Janick J. Loquat: An ancient fruit crop with a promising future. Chronica Hort. 2007:47(2):12-5.

20. Jiang D, Ye QL, Wang FS, Cao L. The mining of citrus EST-SNP and its application in cultivar discrimination. Agri Sci China. 2010;9(2):179-90.

21. Li P, Lin S, Yang X, Hu G, Jiang Y. Molecular phylogeny of Eriobotrya Lindl. (Loquat) inferred from internal transcribed spacer sequences of nuclear ribosome. Pak J Bot. 2009:41(1):185-93.

22. Yang $X$, Najafabadi SK, Shahid MQ, Zhang Z, Jing Y, Wei W, et al. Genetic relationships among Eriobotrya species revealed by genome-wide RAD sequence data. Ecol Evol. 2017;00:1-7.

23. Vilanova S, Badenes ML, Martínez-Calvo J, Llácer G. Analysis of loquat germplasm (Eriobotrya japonica Lindl) by RAPD molecular markers. Euphytica. 2001;121(1):25-9.

24. Soriano JM, Romero C, Vilanova S, Llácer G, Badenes ML. Genetic diversity of loquat (Eriobotrya japonica (Thunb) Lind.) assessed by SSR markers. Genome. 2005;48:108-14.

25. Gisbert AD, Romero C, Martnez-Calvo J, Leida C, Llácer G, Badenes ML. Genetic diversity evaluation of a loquat (Eriobotrya japonica (Thunb) Lindl.) germplasm collection by SSRs and S-allele fragments. Euphytica. 2009;168:121-34.

26. He Q, Li XW, Liang GL, Gao ZS. Genetic diversity and identity of Chinese loquat cultivars/accessions (Eriobotrya japonica) using apple SSR markers. Plant Mol Biol Rep. 2011;29:197-208.
27. Tepe S, Turgutoğlu E, Arslan MA, Polat AA. Improvement of loquat by conventional breeding. Acta Hort. 2010;887:887.

28. Martinez-Calvo BJ, Badenes ML, Llacer G, Bleiholder H, Hack H, Meier U. Phenological growth stages of loquat tree (Eriobotrya japonica (Thunb.) Lindl.). Ann Applied Biol. 1999;134:353-7.

29. Hong $Y$, Lin S, Jiang Y, Ashraf M. The contents of total phenols and flavonoids and antioxidant activity in leaves of 12 Eriobotrya species. Plant Foods Human Nutr. 2008:63:200-4.

30. Liu Y, Song H, Liu Z, Hu G, Lin S. Molecular characterization of loquat EjAP1 gene in relation to flowering. Plant Growth Reg. 2013;70:287-96.

31. Collins FS, Brooks LD, Chakravarti A. A DNA polymorphism discovery resource for research on human genetic variation. Genome Res. 1998;8:1229-31.

32. Li R, Li Y, Fang X, Yang H, Wang J, Kristiansen K. SNP detection for massively parallel whole-genome resequencing. Genome Res. 2009;19:1124-32.

33. Fan B, Du ZQ, Gorbach DM, Rothschild MF. Development and application of high-density SNP arrays in genomic studies of domestic animals. Asian-Aust J Animal Sci. 2010;23(7):833-47.

34. Koopaee HK, Koshkoiyeh AE. SNPs Genotyping technologies and their applications in farm animals breeding programs. Braz Archiv Biol Tech. 2014 57(1):87-95

35. Kumar S, Banks TW, Cloutier S. SNP discovery through next-generation sequencing and its applications. Int J Plant Genomics. 2012;2012:831460

36. Baloch FS, Alsaleh A, Shahid MQ, CËiftcËi V, E SaÂenz de Miera L, Aasim M, et al. A whole genome DArTseq and SNP analysis for genetic diversity assessment in durum wheat from Central Fertile Crescent. PLoS One. 2017; 12(1). e0167821.

37. Henry RJ, Edwards M, Waters DL, Gopala Krishnan S, Bundock P, Sexton TR, et al. Application of large-scale sequencing to marker discovery in plants. J Biosciences. 2012;37:829-41.

38. Xu X, Bai G. Whole-genome resequencing: changing the paradigms of SNP detection, molecular mapping and gene discovery. Mol Breeding. 2015;35:33.

39. Zhang $\mathrm{P}$, Zhong $\mathrm{K}$, Shahid $\mathrm{MQ}$, Tong $\mathrm{H}$. Association analysis in rice: From application to utilization. Front Plant Sci. 2016;7:1202.

40. Miller MR, Dunham JP, Amores A, Cresko WA, Johnson EA. Rapid and costeffective polymorphism identification and genotyping using restriction site associated DNA (RAD) markers. Genome Res. 2007;17(2):240-8.

41. Baird NA, Etter PD, Atwood TS, Currey MC, Shiver AL, Lewis ZA, et al. Rapid SNP discovery and genetic mapping using sequenced RAD markers. PLoS One. 2012;3(10), e3376.

42. Barchi L, Lanteri S, Portis E, Acquadro A, Valè G, Toppino L, et al. Identification of SNP and SSR markers in eggplant using RAD tag sequencing. BMC Genomics. 2011;12:304-12.

43. Hohenlohe PA, Bassham S, Etter PD, Stiffler N, Johnson EA, Cresko WA. Population genomics of parallel adaptation in threespine stickleback using sequenced RAD tags. PLoS Genet. 2010;6(2), e1000862

44. Hohenlohe PA, Amish SJ, Catchen JM, Allendorf FW, Luikart G. Nextgeneration RAD sequencing identifies thousands of SNPs for assessing hybridization between rainbow and westslope cutthroat trout. Mol Ecol Res. 2011;11:117-22.

45. Pfender WF, Saha MC, Johnson EA, Slabaugh MB. Mapping with RAD (restriction-site associated DNA) markers to rapidly identify QTL for stem rust resistance in Lolium perenne. Theor App Genet. 2011;122:1467-80.

46. Takahashi T, Nagata N, Sota T. Application of RAD-based phylogenetics to complex relationships among variously related taxa in a species flock. Mol Phylogenet Evol. 2014:80:137-44.

47. Guo F, Yu H, Tang Z, Jiang X, Wang L, Wang $X$, et al. Construction of a SNPbased high-density genetic map for pummelo using RAD sequencing. Tree Genet Genomes. 2015;11:2.

48. Lin S. Plant material of loquat in Asian countries. First International symposium on loquat, Valencia, Spain, April 2002. Opt Me'diterr. 2004;58:41-4.

49. Tanksley SD, McCouch SR. Seed banks and molecular maps: unlocking genetic potential from the wild. Science. 1997;277:1063-6.

50. Zhou Z, Jiang Y, Wang Z, Gou Z, Lyu J, Li W, et al. Resequencing 302 wild and cultivated accessions identifies genes related to domestication and improvement in soybean. Nature Biotech. 2015;33:408-16.

51. Zhang Z, Mao L, Chen H, Bu F, Li G, Sun J, et al. Genome-wide mapping of structural variations reveals a copy number variant that determines reproductive morphology in cucumber. Plant Cell. 2015;27(6):1595-604.

52. Cao K, Zheng Z, Wang L, Liu X, Zhu G. Comparative population genomics reveals the domestication history of the peach, Prunus persica, and human influences on perennial fruit crops. Genome Biol. 2014;15:415. 
53. Aflitos SA, Schijlen E, Finkers R, Smit S, Wang J, Zhang G, et al. Exploring genetic variation in the tomato (Solanum section Lycopersicon) clade by whole-genome sequencing. Plant J. 2014;80(1):136-48.

54. Mylesa S, Boyko AR, Owens CL, Cresko WA, Johnson EA. Genetic structure and domestication history of the grape. Proc Natl Acad Sci U S A. 2011; 108(9):3530-5.

55. Zhang HZ, Zhang YD. A study on the native loquats in Hubei province. J Huazhong Agri. 1982;03:86-93.

56. Zhang HZ, Peng SA, Cai LH, Fang QD. The germplasm resources of the genus Eriobotrya with special reference on the origin of E. japonica Lindl. Acta Hort Sin. 1992;17(1):2-12.

57. Riley TJ, Edging R, Rossen J. Cultigens in prehistoric eastern North America: changing paradigms. Curr Anthropol. 1990;31:525-41.

58. Blumer MA. Independent inventionism and recent genetic-evidence on plant domestication. Econo Bot. 1992;46:98-111.

59. Zohary D. Monophyletic vs. polyphyletic origin of crops on which agriculture was founded in the near East. Genet Res Crop Evol. 1999:46:133-42.

60. Heun M, Schäfer-Pregl R, Klawan D, Castagna R, Accerbi M, Borghi B. Site of einkorn wheat domestication identified by DNA fingerprinting. Science. 2007;278:1312-4.

61. Spooner DM, McLean K, Ramsay G, Waugh R, Bryan GJ. A single domestication for potato based on multilocus amplified fragment length polymorphism genotyping. Proc Natl Acad Sci U S A. 2005;102:14694-9.

62. Matsuoka Y, Vigouroux Y, Goodman MM, Sanchez G. A single domestication for maize shown by multilocus microsatellite genotyping. Proc Natl Acad Sci U S A. 2002:99:6080-4.

63. Qi J, Liu X, Shen D, Miao H, Miao H, Xie B, Li X, et al. A genomic variation map provides insights into the genetic basis of cucumber domestication and diversity. Nat Genet. 2013;45(12):1510-8.

64. Schmutz J, McClean PE, Mamidi S, Wu GA, Cannon SB, Grimwood J, et al. A reference genome for common bean and genome-wide analysis of dual domestications. Nat Genet. 2014;46(7):707-13.

65. Pourkheirandish M, Hensel G, Kilian B, Senthil N, Chen G, et al. Evolution of the grain dispersal system in barley. Cell. 2015;162:527-39.

66. Liu Q, Wang GR, Rv JL, Shen DX. Quantitative classification of loquat cultivars resource. Fruit Sci. 1993;10(3):137-41.

67. Ding CK, Chen QF, Sun TL, Xia QZ, Zhu DW. Germplasm resources and breeding of Eryobotria japonica Lindl. in China. Acta Hort. 1995;403:121-6.

68. Doyle JJ, Doyle JL. A rapid DNA isolation procedure for small quantities of fresh leaf tissue. Phytochem Bull. 1987;19:11-5.

69. Etter PD, Preston JL, Bassham S, Cresko WA, Johnson EA. Local De Novo assembly of RAD paired-end contigs using short sequencing reads. Plos One. 2011;6(4), e18561.

70. Catchen J, Amores A, Hohenlohe P, Cresko W, Postlethwait J. Stacks: building and genotyping loci de novo from short-read sequences. G3: Genes Genomes Genet. 2011;1:171-82.

71. Excoffier L, Laval G, Schneider S. Arlequin ver. 3.0: An integrated software package for population genetics data analysis. Evol Bioinformatics Online. 2005;: :47-50.

72. Saitou N, Nei M. The neighbor-joining method: A new method for reconstructing phylogenetic trees. Mol Biol Evol. 1987;4:406-25.

73. Nei M, Kumar S. Molecular evolution and phylogenetics. New York: Oxford University Press; 2000.

74. Tamura K, Stecher G, Peterson D, Filipski A, Kumar S. MEGA6: Molecular evolutionary genetics analysis version 6.0. Mol Biol Evol. 2013:30:2725-9.

75. Price AL, Patterson NJ, Plenge RM, Weinblatt ME, Shadick NA, Reich D. Principal components analysis corrects for stratification in genome-wide association studies. Nat Genet. 2006;38:904-9.

76. Pritchard JK, Stephens M, Donnelly P. Inference of population structure using multilocus genotype data. Genetics. 2000;155:945-59.

\section{Submit your next manuscript to BioMed Central and we will help you at every step:}

- We accept pre-submission inquiries

- Our selector tool helps you to find the most relevant journal

- We provide round the clock customer support

- Convenient online submission

- Thorough peer review

- Inclusion in PubMed and all major indexing services

- Maximum visibility for your research

Submit your manuscript at www.biomedcentral.com/submit
C Biomed Central 